\title{
DIFFICULTY IN FIXATION OF FRACTURE NECK OF FEMUR IN A PARALYTIC HIP: A CASE REPORT
}

\author{
S. Suresh Babu¹, T. V. Suresh Babu², Kopuri Ravi Kiran³, G. Sudhakar4, M. U. S. K. Sridevi ${ }^{5}$
}

\section{HOW TO CITE THIS ARTICLE:}

S. Suresh Babu, T. V. Suresh Babu, Kopuri Ravi Kiran, G. Sudhakar, M. U. S. K. Sridevi. "Difficulty in Fixation of Fracture Neck of Femur in a Paralytic Hip: A Case Report". Journal of Evolution of Medical and Dental Sciences 2014; Vol. 3, Issue 30, July 28; Page: 8484-8489, DOI: 10.14260/jemds/2014/3074

ABSTRACT: Fracture neck of femur is known for its difficult in closed fixation, delayed union, nonunion and avascular necrosis if presented late to hospital. We report a case of Fracture neck of Femur in a paralytic hip having difficulty in closed fixation due to excessive anteversion of 58 degrees, valgus neck of 138 degrees, small neck, dislocated and dysplastic hip presented 18 days after an injury.

KEYWORDS: Difficult fixation, fracture neck of femur, paralytic hip.

INTRODUCTION: Fracture neck of Femur in a paralytic hip will have difficulty in closed fixation due to excessive anteversion, valgus neck, small neck, dislocated and dysplastic hip. We need to anticipate and approach before taking an incision. Incidence of fracture neck of femur in post-polio residual paralysis is not much available in literature. People with post-polio residual paralysis are prone to fractures after mild trauma. The flaccid paralysis, asymmetric involvement, and underdeveloped growth of affected leg may lead to osteoporosis is the common cause to develop fracture neck femur.

CASE REPORT: 28 year old man having post-polio residual paralysis admitted in our hospital 18 days following a trivial injury with pain around right hip. He used to walk with hand to knee gait before injury independently. He was having apparently normal upper limbs with fairly good muscular power in other lower limb. Patient was clinically normal for other systems. Radiographs taken in both neutral and internal rotation of hip showed garden stag 4 fracture neck of femur, osteoporosis, valgus neck, dysplastic and dislocated hip (figure 1).

Hip was reduced under spinal anesthesia on fracture table using c-arm by traction flexion abduction and internal rotation maneuver. It was observed that we were able reduce the fracture in excessive internal rotation of hip around more than 50 degrees, abduction of 30 degrees which was unusual for fracture neck of femur. We failed in passing guide wire in the centre of neck in both anteroposterior and lateral view through percutaneous incision of $1 \mathrm{~cm}$ below greater trochanter.

Then we took a stab incision more posteriorly almost in the buttock and passed guide wire in middle of the neck due to high anteversion (figure 2). Fracture was fixed and compressed with three $32 \mathrm{~mm}, 6.5 \mathrm{cc}$ threaded cannulated cancellous screws. Triangular configuration of screws was not possible due to small neck and difficulty in fixation while performing surgery in an awkward position. Patient was advised non weight bearing for 6 months and was followed with regular radiographs till fracture was united (figure 3).

Retrospectively we analyzed the hip by clinical, radiological and CT scan after fracture union. Anteroposterior radiograph of hip was taken with hip neutral rotation which showed the united fracture neck of the femur as an end on appearance. When hip was internally rotated of more than 50 degrees, anteroposterior radiograph was able to show the entire neck in full length. That means clinically patient is having excessive anteversion of 50 degrees (figure 4, 5). 
CT scan was taken in both neutral and internal rotation to measure femoral neck anteversion. It was measured that the anteversion ( $\mathrm{NC}=$ neck condyle) was 58.835 degrees in neutral rotation (NC58.835= NH76.994-CH18.159). In internal rotation anteversion was 58.704 (NC58.704= NH32.098+CH26.606) (figure 6, 7). After 2 years patient was walking pain free and without avascular changes.

DISCUSSION: More than 250000 hip fractures occur in the United States each year and this number is projected double by the year $2050 .{ }^{1}$ The average age of occurrence of femoral neck fracture is 77 years for women and 72 years for men due to osteoporosis. ${ }^{2}$ Femoral neck fractures in young adults are considered as a separate group because they occur in normal bone and are uncommon. These fractures are high shear type fractures that extend near the lesser trochanter with high incidence of delayed union, non-union, avascular necrosis different from elderly. ${ }^{3}$

Incidence of fracture neck of femur in post-polio residual paralysis is not much available in literature. People with post-polio residual paralysis are prone to fractures after mild trauma. The flaccid paralysis, asymmetric involvement, and underdeveloped growth of affected leg may lead to osteoporosis. Chang et al studied 32 men by their femoral BMD and found osteoporosis to be the causative factor for these fractures. ${ }^{4}$ Ausif in his study of 51 patients found 8 of 9 patients with trivial trauma had fracture neck of femur. ${ }^{5}$ Our patient is also a known case of post-polio residual paralysis with radiological osteoporosis presented 18 days after a trivial injury.

Of the various methods of treating a fracture neck of femur, the standard method for treating a young patient is closed reduction and 6.5 cannulated screw fixation, if closed reduction fails open reduction is mandatory. ${ }^{6}$ Meyers used muscle pedicle graft in 23 patients less than 40 years of age. ${ }^{7}$

Swiontkowski presented 27 patients between 12 and 49 years of age were treated with $6.5 \mathrm{cc}$ screw fixation within 8 hours of injury, all fractures were united with $20 \%$ avascular necrosis. ${ }^{8}$ In our patient reduction was achieved by gross internal rotation of more than 50 degrees, difficulty in passing guide wire in centre of neck was due to excessive anteversion which is unusual.

Later a posterior entry point close to the buttock was taken to negotiate the guide wire in the centre of the neck. Post operatively we analysed that our difficulty was due to excessive anteversion (50 degrees), excessive valgus neck of around 138 degrees and unstable paralytic hip. Probably because of excessive valgus the reduction was stable for fixation and went on union even after delayed presentation.

Femoral version is defined as the angular difference between axis of femoral neck and transcondylar axis of the knee; on average, femoral anteversion ranges from 30-40 deg at birth and decreases throughout growth to about 15 deg at skeletal maturation; in adults, anteversion averages between 8 and $14 \mathrm{deg}$, an average of 8 degrees in men and 14 degrees in women. Any increase or decrease in the angle of femoral anteversion is associated with various clinical conditions.

The increased angle of anteversion is associated with $\mathrm{CDH}$, Perthes, slipped epiphysis, cerebral palsy, poliomyelitis, postural defects, squinting patellae, apparent genu valgum, tibial extorsion, flat foot, and in toeing. ${ }^{9,10}$

The decreased femoral torsion has been shown with toeing out, rickets, and chondrodystrophy. Researchers worldwide have used various methods and stated CT-Scan is most reliable method. In CT-scan anteversion is measured as neck condylar angle (NC) =neck horizontal angle $(\mathrm{NH})$ - condylar horizontal angle $(\mathrm{CH})$ in neutral rotation of hip, or in internal rotation of hip 
measured as neck condylar angle (NC) =neck horizontal angle $(\mathrm{NH})+$ condylar horizontal angle (CH). ${ }^{11}$ Our patient after fracture union was analysed clinico-radiologically and by CT scan in both neutral and internal rotation of hip.

It was observed that the full neck length was seen in follow-up radiographs showing union, radiograph that was taken in internal rotation of more than 50 degrees of hip. This infers that patient was having an anteversion of more than 50 degrees.

CT scan showed patient had an average anteversion of 58degrees in both neutral and internal rotation. This was confirmed to be the cause for difficulty intraoperatively in passing the guide wire in the centre of neck through mid-lateral. Patient was absolutely symptom free at 2 years follow-up

\section{REFERENCES:}

1. Koval K J, Zuckerman J D. Functional Recovery after hip fracture. J Bone Joint Surgery1994; 77: 751-758.

2. AAOS Bulletin. Femoral neck fracture (Adult), pp. 11-12.Chicogo, 11, 1989.

3. Kulisch S D, Gustilo R B. Fractures of the femoral neck in Young adults. J Bone Joint Surg1976; 58A: 724.

4. Chang $\mathrm{KH}$, Lai $\mathrm{CH}$, Chen SC, et al. Femoral neck bone mineral density in ambulatory men with poliomyelitis. Osteoporos Int. 2011 Jan; 22(1):195-200.

5. Ausaf F. High incidence of osteoporosis and fractures in an aging post-polio population. Eur Neurology. 2009; 62 (6): 369-74.

6. Cave E F. Fracture of the femoral neck. Instr Course Lecture1960; 17: 79-93.

7. Meyers M H. The role of posterior bone Grafts (Muscle Pedicle) in Femoral neck fractures. Clinical. Orthop1980; 152: 143-146.

8. Swiontkowski M F. Fractures of the femoral neck in patients between the ages of twelve and forty nine years. J Bone Joint Surg1984; 66A:837-846.

9. Upadhyay SS, Burwell RG, Moulton A et al. Femoral Anteversion in healthy children: application of a new method using ultrasound. J Anat 1987; 169: 49-61.

10. Kirby AS, Wllace WA, Moulton A et al. Comparison of four methods for measuring femoral anteversion. Clinical Anatomy 1993; 6:280-288.

11. Visions/MSK/Protocols revised 2/16/04 @ Ken L Schreibman, PhD/MD www.radiology.wisc.edu/Di UW CT Protocol: FEMORAL ANTEVERSION.

Fig.1: Pre-op, antero-Posterior radiographs in both neutral and internal rotation.

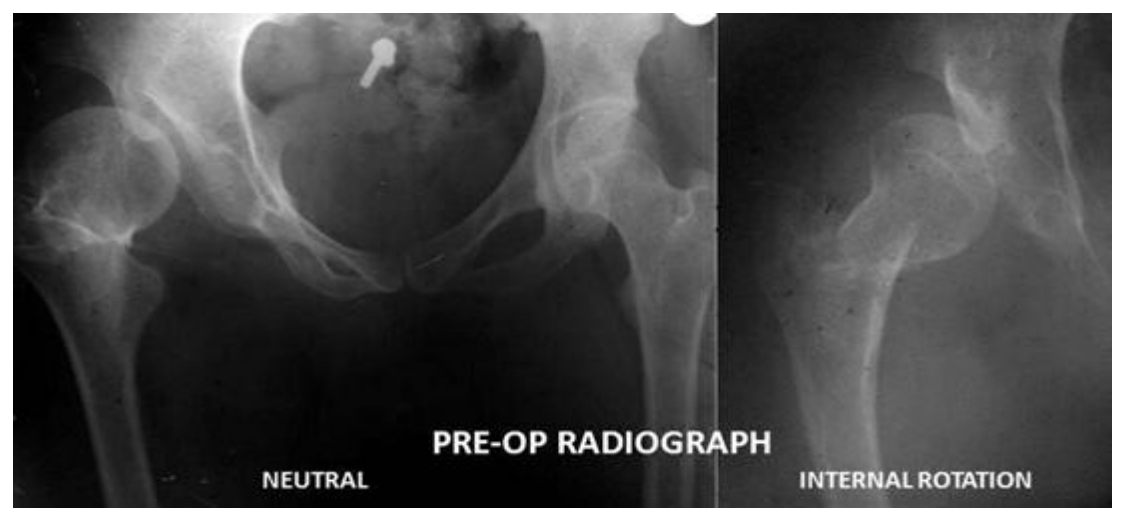

Fig. 1 


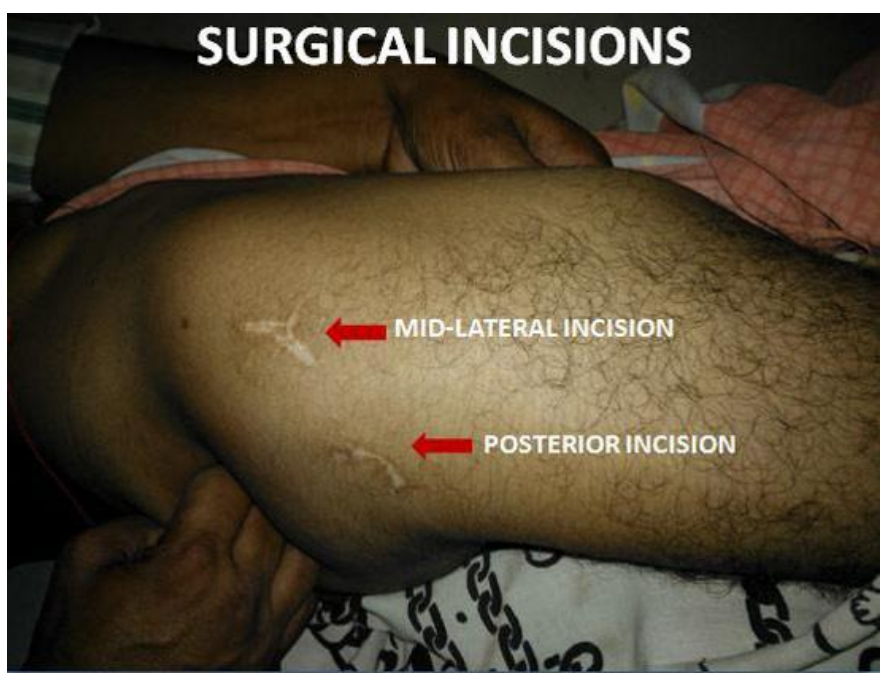

Fig. 2: Surgical Incisions

Fig. 3: Post-op, radiographs Showing Immediate and 5 months follow up.

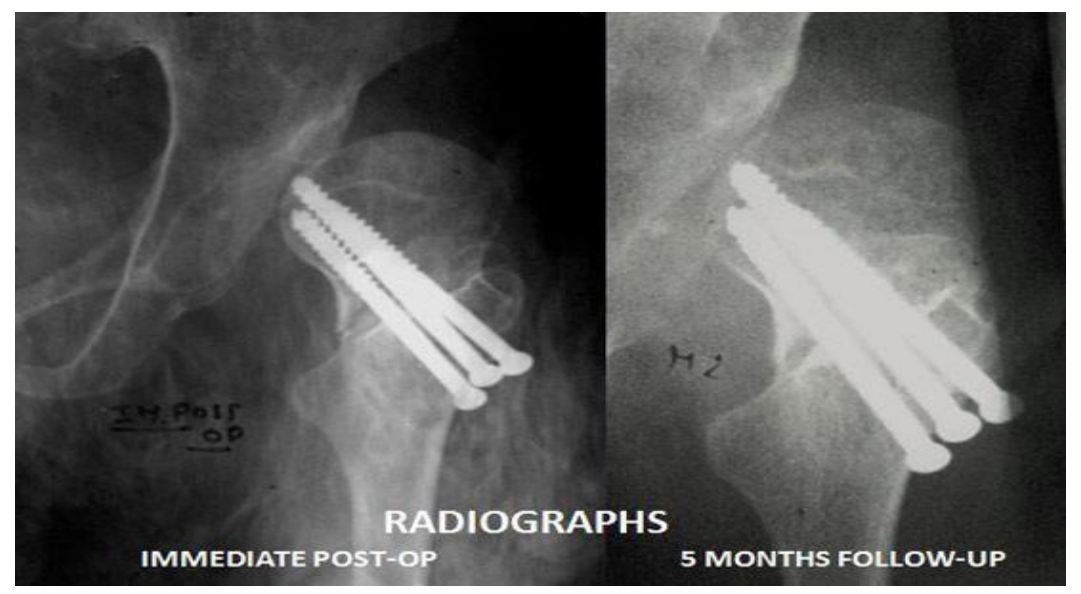

Fig. 3 
Fig. 4: Clinical Photograph and corresponding radiograph of the patient hip in neutral rotation at 2 year follow up.

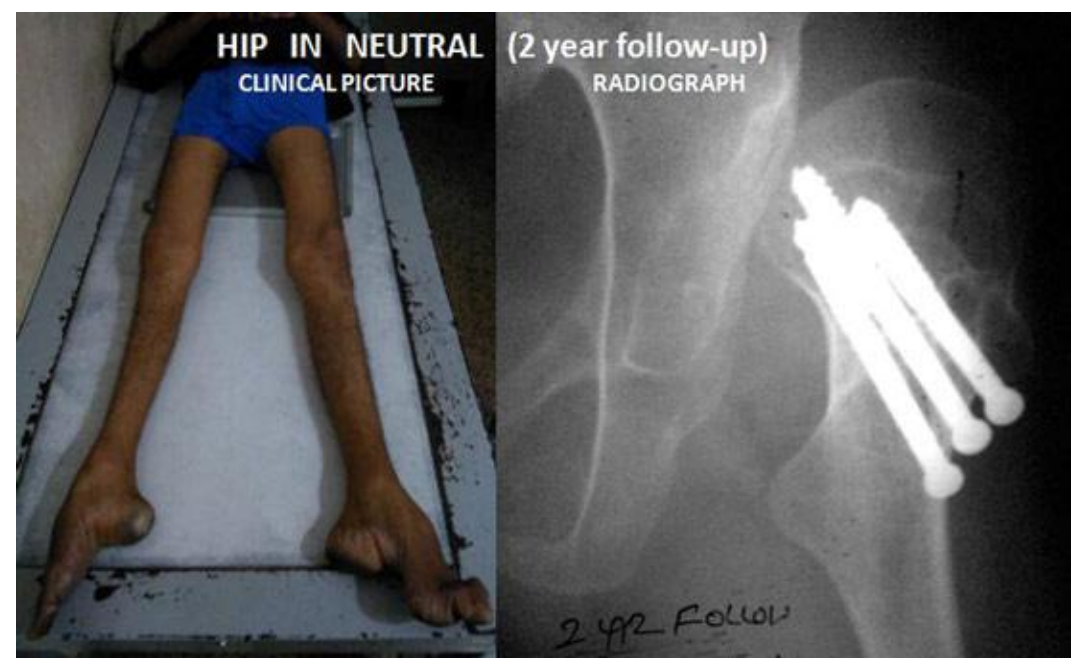

Fig. 4

Fig. 5: Clinical Photograph and corresponding radiograph of the patient hip in internal rotation at 2 year follow up.

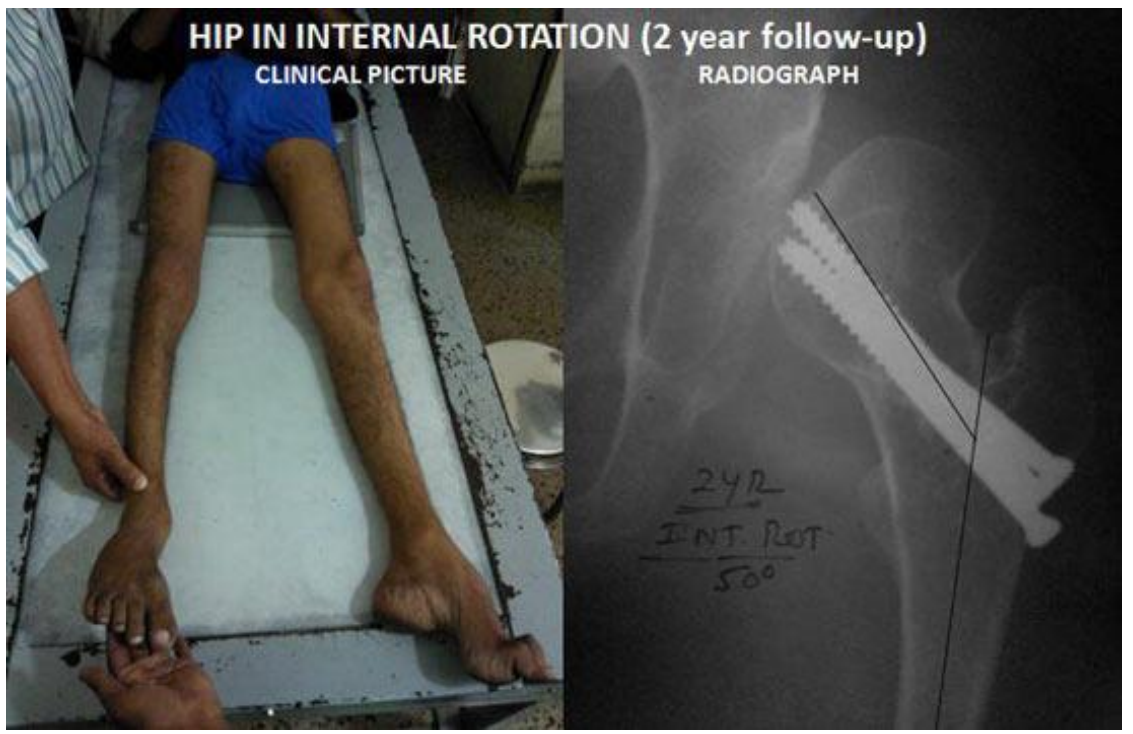

Fig. 5 


\section{CASE REPORT}

Fig. 6: CT-scan of the hip in neutral rotation showing angle of anteversion of 58.8 degrees.

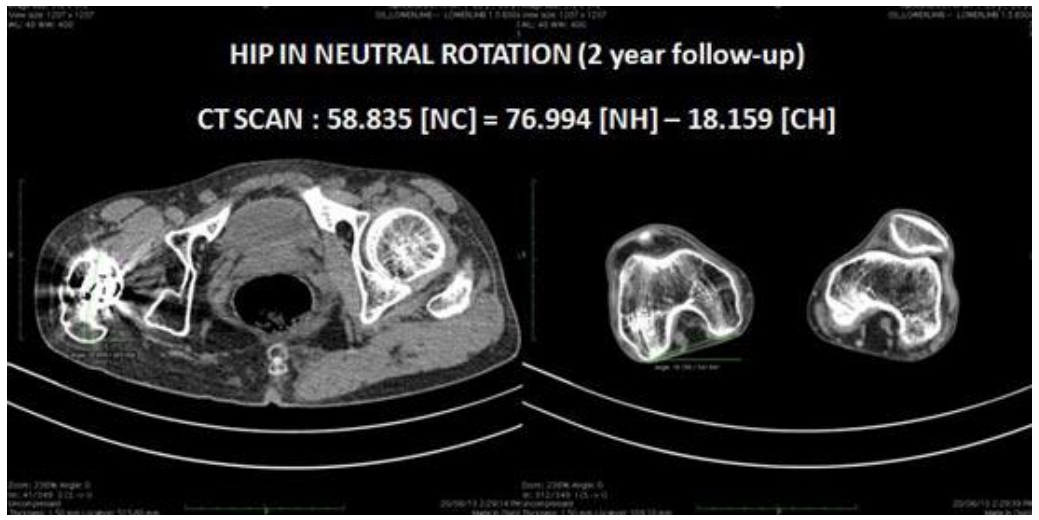

Fig. 6

Fig.7: CT-scan of the hip in internal rotation showing angle of anteversion of 58.7 degrees.

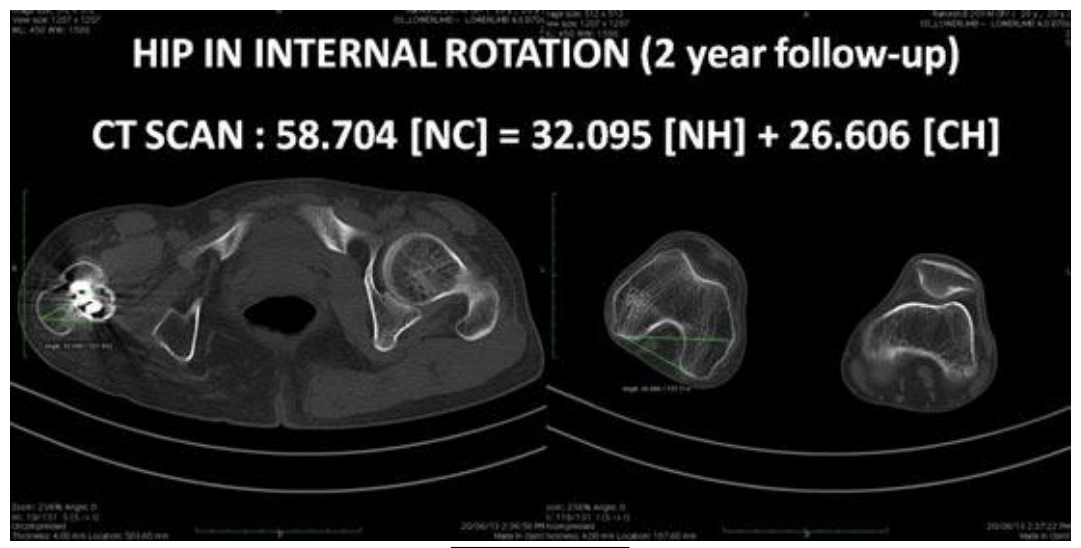

Fig. 7

\section{AUTHORS:}

1. S. Suresh Babu

2. T. V. Suresh Babu

3. Kopuri Ravi Kiran

4. G. Sudhakar

5. M. U. S. K. Sridevi

\section{PARTICULARS OF CONTRIBUTORS:}

1. Professor, Department of Orthopaedics, Dr. PSIMS \& RF.

2. Associate Professor, Department of Orthopaedics, Dr. PSIMS \& RF.

3. Associate Professor, Department of Orthopaedics, Dr. PSIMS \& RF.

4. Assistant Professor, Department of Pathology, Siddhartha Medical College.

5. Anaesthesiologist Consultant, Department of Anaesthesia, Suresh Ortho Clinic.

\section{NAME ADDRESS EMAIL ID OF THE} CORRESPONDING AUTHOR:

Dr. S. Suresh Babu,

Professor, Department of Orthopedics, Dr. PSIMS \& RF, Suresh Ortho Clinic, \#56-2-20, Koneru Satyanarayana Street, Patamata, Vijayawada-520010, Krishna District, India.

Email: drssureshbabu@yahoo.com

Date of Submission: 27/06/2014. Date of Peer Review: 28/06/2014. Date of Acceptance: 15/07/2014. Date of Publishing: 25/07/2014. 